\title{
A LA MEMORIA DE CARLOS LENKERSDORF (1926-2010)
}

\author{
ye'n wa sje'a ki'tik / ja kermanotik / karlos sb'i'ili / ti b'a chonab'i. // ja \\ ye'n jel lek / jel lek ja sk'ujol / wa xyala kab'tik / ja jlekilaltik. \\ El educador / nuestro hermano es / Carlos es aquél / y es de \\ Comitán. // Buena gente es él / bueno su pensar / nos explica cuál \\ / es la libertad \\ "Kurso b’a tililte" ["Curso en Tililté"], Indios somos con orgullo. \\ Poesía maya-tojolabal, 74 y 75
}

La noche del 23 de noviembre de 2010, el teólogo, doctor en filosofía y lingüista práctico Karl Heinz Herman Lenkersdorf Schmidt, sólo Carlos para sus queridos hermanos tojolabales, amigos, colegas y alumnos, alcanzó el punto final de los sufrimientos corporales y encontró la paz completa. Sin embargo, su palabra de expresión sencilla pero inspiradora no ha sido silenciada con el trivial acto de morir. En manos de quienes lo siguen admirando, la mayoría de ellos jóvenes universitarios, estudiantes de las humanidades, quedaron sus libros. Textos desde donde su apasionada convicción indigenista seguirá interpelando a los escépticos y detractores que, con base en la antropología, la linguística o la historia, niegan la posibilidad de la existencia de una comunidad limpia de las lacras que él consideraba herencia del individualismo de la sociedad occidental.

Si se acepta el principio formulado por el notable ex alumno de la Universidad de Marburgo, José Ortega y Gasset, de que el hombre es él y su circunstancia, al tratar de comprender cómo Carlos llegó a convertirse en el intérprete, representante y propagandista de una etnia minoritaria entre las mayanses pobladoras del estado de Chiapas, resulta necesario detenerse a considerar, tanto las peculiares características de su preparación académica, como lo variado, rico y contrastante de sus experiencias vitales.

La segunda década del siglo xx, tras la derrota en la Gran Guerra y frente a las exigencias del tratado de Versalles, se caracterizó en la historia de Alemania por el enorme esfuerzo de su diezmada población para lograr la reconstrucción económica y la estabilidad política, bajo el régimen de la República de Weimar. Fue en el año de 1926, el 7 de agosto, cuando Karl vio la luz en Berlín, como hijo segundo y último del dueño de una panadería. Liquidada la república, sus primeros años de escolar transcurrieron a lo largo de la etapa en que Hitler logró imponer la dictadura nacional socialista. Entonces aprendió el noble oficio paterno y desarrolló el gusto por la música clásica que lo acompañó toda su vida. Ya octogenario, recordaría con tristeza la desaparición de su profesor de piano, arrestado durante alguna redada decretada contra los judíos. Karl entró a 
la adolescencia en días marcados por el militarismo y el racismo, cuando la victoriosa marcha del ejército logró la expansión del territorio alemán más allá de las fronteras asignadas por los vencedores en 1919 y cuando desde el gobierno fue planeado el exterminio de la raza aborrecida.

La guerra se materializó frente a las puertas del hogar de los Lenkersdorf menos de 20 días después del cumpleaños número 14 de Karl, en agosto de 1940, con los bombardeos de la aviación británica que, al paso del tiempo, dejaron su ciudad natal reducida a escombros. Al cumplir los 16 le tocó dejar la escuela para seguir a su hermano en el cumplimiento del servicio militar obligatorio. Muy pronto el primogénito murió en el frente oriental polaco y Karl partió con un grupo de estudiantes a reforzar las defensas del Rin. De aquellos días recordaba cómo su superior les había asignado distintas tareas tras preguntarles cuál era la ocupación de sus padres. Así el recluta fue destinado a la cocina. Ante la imposibilidad de detener la ofensiva lanzada por los aliados en la primavera de 1945, el comandante, seguro del desastre inminente y con el fin de evitar que los muchachos cayeran prisioneros, los dio por libres para que regresaran ocultos, dispersos y por sus propios medios a sus lugares de origen. Entonces Karl emprendió caminando solo el largo regreso a casa. Todavía lejos de Berlín, donde ya se combatía calle por calle la última batalla contra los soviéticos, y merced a la compasión de una señora encontró alimento y refugio, mientras buscaba la manera de comunicarse con sus padres. Al viejo Karl, por obvias razones, no le gustaba conversar sobre los sucesos de la contienda; pero resulta imposible soslayar el impacto que debe haber tenido en el desarrollo de su personalidad y la formación de su pensamiento el hecho de haber alcanzado el estatus de veterano de guerra apenas a los dieciocho años, en el corazón de una Alemania a la que había aprendido a venerar como una patria grande y poderosa, ahora derrotada, ocupada y dividida por los extranjeros.

Recobradas, aunque en forma muy precaria, las condiciones de normalidad para la población, en abril de 1946 Karl concluyó el bachillerato en Hannover y, desechada la idea de convertirse en músico, tomó la decisión de matricularse en la carrera de teología, en momentos cuando resultaba penosa en extremo la situación de los sobrevivientes. Entonces ingresó a uno de los seminarios protestantes más prestigiados del mundo, entre otras cosas por el rigor del análisis filológico al que sus catedráticos sometían los textos bíblicos, la Divinity School de la Philipps-Universitat de Marburgo. Allí fue discípulo de Rudolf K. Bultmann. El teólogo que propuso desmitificar el Evangelio para volver su esencia humanista accesible al hombre contemporáneo. Estos estudios los terminó en la Evangelisch-Theologische Fakultät de la Universidad de Bonn, con la defensa de la tesis: "La justitia dei en el comentario de Martín Lutero a la carta de san Pablo a los romanos", el 28 de julio de 1951.

Apenas instalado en Marburgo, el estudiante conoció a Gudrun Hildegard Lohmeyer Linder, una joven recién llegada de Bremen para cursar la carrera de física. Seis décadas más tarde, Karl recordaba sonriente lo nervioso que se había sen- 
tido al enfrentar, durante su primera exposición pública, a un auditorio formado por muchachas, entre las cuales además se encontraba Gudrun, su condiscípula de los cursos de historia medieval que siempre era tan crítica. En 1952, ella se convirtió en la esposa y fiel compañera con la que compartiría el resto de su vida. La oferta de una beca para realizar el doctorado en la Universidad de Chicago le abrió la posibilidad de trasladarse al Nuevo Mundo. Al año siguiente, por el puerto de Bremen, primero Karl y meses después Gudrun, que no pudo partir hasta titularse, salieron de la apenas fundada República Federal de Alemania, y como tantos otros inmigrantes europeos, no sin sufrir las incomodidades propias de la navegación, fueron recibidos por la Estatua de la Libertad en Nueva York.

Poco más de un año duró aquella primera estancia de los Lenkersdorf en los Estados Unidos; entre otras causas de disgusto, sobresalen los desacuerdos del doctorando con sus nuevos profesores, teólogos dogmáticos, y la desconfianza de la joven científica respecto de la finalidad, sospechosamente belicista, de las investigaciones realizadas en el Departamento de Física, donde había empezado a trabajar. Entonces la pareja optó por mudarse a Canadá. De una urbe moderna y cosmopolita como Chicago pasaron a vivir en la población rural de Oliver, en un valle de la montañosa Columbia Británica, habitada por agricultores evangélicos, descendientes de los alemanes del Volga, austeros, muy trabajadores y ultraconservadores. Allí Karl ejerció la labor pastoral como ministro de la Iglesia Luterana durante los años siguientes.

En busca de nuevos horizontes, en 1957 los Lenkersdorf se trasladaron a la ciudad de México, en esa década la capital en crecimiento de un país en pleno desarrollo económico, a donde Karl había sido convocado para dar clases de griego en el Centro Augsburgo, establecido en Coyoacán por la misma Iglesia y del que llegó a ser rector, aunque sus colegas lo tachaban de heterodoxo. Entonces con disciplina y ahínco ambos se aplicaron al aprendizaje del español. Durante las vacaciones de la primavera del año siguiente, la pareja tuvo un encuentro circunstancial con el mundo de los mayas, cuando transitaron por Veracruz y Tabasco hasta el todavía selvático Palenque, y luego por Campeche rumbo a Mérida para visitar Uxmal y Chichén Itzá. Estos viajeros todavía estaban lejos de imaginar la importancia que dichos pueblos indígenas llegarían a alcanzar, más adelante, en el transcurso de sus existencias.

En 1960, Karl publicó en la editorial religiosa El Escudo su primer libro, un Comentario sobre la epístola a los gálatas. Texto redactado ya en español, en cuyas líneas volvía a reflexionar sobre el tema de las enseñanzas de Pablo de Tarso, que habían sido motivo de la exégesis de Lutero.

A principios del año siguiente, con el propósito de comprender el país donde ahora residía y de diversificar las actividades académicas en que podía ocuparse, Karl ingresó a la Universidad Nacional Autónoma de México para cursar la carrera de filosofía. Cuatro años después se tituló de la licenciatura con la defensa de la tesis: "Epicteto, su metafísica en relación con la ética", el 23 de octubre de 1964. Un estudio dedicado a este filosofo estoico griego del siglo I, que sufrió 
el exilio y la esclavitud en Roma, y es en la actualidad reconocido por sus reflexiones sobre moral. Luego, obtuvo su doctorado el 4 de noviembre de 1966 con la tesis: "El concepto del trabajo en el cristianismo primitivo". De aquella su segunda época como estudiante, en una facultad donde se cultivaba la postura crítica frente a las corrientes del pensamiento llegadas de Europa, durante una década caracterizada por los ideales libertarios de los jóvenes y el deseo compartido entre muchos maestros y alumnos de tener una participación activa en la transformación de la sociedad, a la memoria de Karl acudían recuerdos sobre tres de sus profesores, todos miembros del grupo de intelectuales exiliados a raíz de la derrota de la República Española, que tantas aportaciones legó a las humanidades en México: el notable exponente de la reflexión ética Eduardo Nicol, el marxista Adolfo Sánchez Vázquez (autor de la Filosofía de la praxis, un libro guía para la acción social), y el director de su tesis doctoral Wenceslao Roces, militante comunista y profundo conocedor, traductor e intérprete de las obras fundamentales de Marx y Engels.

La primera publicación del doctor en filosofía apareció en 1968. Se trata del capítulo: "Religión y revolución" en el libro colectivo: La Iglesia, el subdesarrollo y la revolución, impreso por la editorial Nuestro Tiempo. Volumen donde comparte créditos con el etnólogo dominico Tomás G. Allaz, el politólogo Víctor Flores Olea, el sociólogo Horacio Labastida y el obispo de Cuernavaca Sergio Méndez Arceo, entre otros. Todos pensadores que expusieron su enfoque desde la Iglesia Católica, el periodismo o la academia acerca del problema de la justicia social, en el contexto latinoamericano de los países agrupados bajo el concepto del Tercer Mundo y de la preocupación por los pobres expresada en el Concilio Vaticano II.

Al mismo tiempo que realizaba su doctorado, Karl trabajó como catedrático de filosofía en la Universidad de las Américas, institución heredera del México City College, donde ya graduado obtuvo el nombramiento de decano académico de humanidades, entre 1967 y 1969. (Los mismos dos años en que se desempeñó como maestro de filosofía de la religión y de ética en la Facultad de Filosofía y Letras de la unam.) Sin ánimo para permanecer en la ciudad de México después de la represión del movimiento estudiantil del 68, los Lenkersdorf, ahora acompañados por sus dos hijos, partieron de nuevo a los Estados Unidos. Regresaron a la potencia capitalista, porque Karl fue nombrado director de Estudios Latinoamericanos en la Antioch College en Yellow Springs, Ohio. Ese puesto le permitió viajar a distintos países como Puerto Rico, Colombia, el Chile socialista gobernado por Salvador Allende y a Ecuador, donde tuvo otro acercamiento al mundo campesino indígena al conocer las escuelas rurales para los quechuas. Entonces escribió el artículo "Iglesia y liberación del pueblo", aparecido en 1972 en Montevideo, Uruguay, como parte de la obra colectiva: Pueblo oprimido, señor de la historia, cuyo título evoca, con la imagen de la esclavitud de los israelitas en Egipto, uno de los temas predilectos de la Teología de la Liberación.

Ese mismo año, durante una estancia en México, conoció al obispo Samuel Ruiz. Aquel encuentro, propiciado por un amigo mutuo, definió su futuro. Tras 
una larga conversación los dos cristianos, exegetas del Evangelio, se identificaron en el anhelo de coadyuvar al cambio de la sociedad. Entonces el ministro luterano y filósofo marxista aceptó la invitación del prelado católico para visitar Chiapas aquel verano, con el propósito de considerar la posibilidad de integrarse como colaborador del proyecto diocesano de organización de la Iglesia autóctona. Un proyecto construido sobre los postulados del Concilio Vaticano II y las resoluciones del Consejo Episcopal Latinoamericano celebrado en Medellín, Colombia, en 1968. A partir de este histórico sínodo, las determinaciones conciliares desbordaron los cauces reformistas propuestos por Juan XXIII, para correr por las vías revolucionarias de la teología de la liberación como base de una Iglesia comprometida con las causas de los desheredados y oprimidos, en busca del establecimiento de la justicia social en este mundo.

En la misión jesuita de Bachajón, pueblo de los altos, Karl tuvo su primera aproximación con hablantes de la familia linguiística mayanse durante una asamblea de representantes de diferentes comunidades tzeltales. En medio de la tan animada como prolongada discusión en el idioma desconocido, distinguió sin entender la repetición del sufijo -tik, equivalente a "nosotros". Concepto que con el tiempo interpretaría como distintivo de la cultura maya y del que afirmaría ser la clave para comprenderla desde dentro.

Entusiasmado con la posibilidad de participar en un proyecto de transformación social, Karl sólo regresó a Ohio para ponerse de acuerdo con su esposa y renunciar al nombramiento académico que allí tenía. Con el completo apoyo de Gudrun, que también dejó el puesto que ocupaba como docente en la misma institución educativa, la familia cambió su residencia a Comitán. Desde 1973, ambos laboraron en La Castalia, Escuela de Promoción Educativa y Cultural para los Indígenas de la Región Tojolabal. Aunque el grupo de trabajo donde se integraron no lo consideraba necesario, por el grado de castellanización de los indígenas, ellos decidieron que lo primero para poder entenderse con la población a la que debían alfabetizar era aprender su lengua. Entonces solicitaron a los tojolabales les auxiliaran enviándoles alguien que les enseñara. Por acuerdo de la comunidad se atendió esta petición y se designó a los comuneros que, por turnos, se encargarían de instruir a los recién llegados. Como los tojolabales, además de ser mayoritariamente analfabetos, eran ágrafos en su propio idioma, fueron los alumnos quienes empezaron a poner por escrito, frente a los instructores, lo que aprendían; este fue un acto que se convirtió en demostración evidente, tanto para los hablantes como para los comitecos, de que la lengua tojolabal también podía expresarse mediante el alfabeto. Aquel fue el primer paso, calificado por Karl de "diálogo intercultural", que lo llevó a la investigación linguiística y a elaborar su propia interpretación de la cultura autóctona, enriquecida a lo largo del tiempo como resultado de la vivencia cotidiana en las comunidades de los municipios de las Margaritas y de Altamirano.

Entre 1973 y 1975 (años marcados en el estado de Chiapas por la organización, desarrollo y consecuencias del primer Congreso Indígena, celebrado en 
honor de fray Bartolomé de las Casas en la capital del estado), Karl coordinó la elaboración y la Escuela publicó los libros de texto en tojolabal para la alfabetización y la enseñanza de la aritmética, entre otros escritos de orientación sobre derechos agrarios y garantías individuales, además de creación literaria. En 1976 empezó la composición del Diccionario tojolabal-español, que en su primera versión fue publicada en 1979 por la editorial Nuestro Tiempo. Luego, en 1981 vio la luz el Diccionario español-tojolabal, también estampado por la misma editorial. Obras que después de reimprimirse se reeditaron en versiones corregidas y aumentadas, la primera en el 2004 y la segunda en 1996.

En el ir y venir de una comunidad a otra y con el afán de profundizar en el trasfondo filosófico de la lengua, para lograr lo que consideraba la verdadera comprensión de la cultura maya; Karl, como le había ocurrido a Saulo de Tarso, encontró por aquellas veredas, polvorientas bajo el sol o lodosas bajo la lluvia, su propio camino de Damasco. Sufrió un proceso de conversión, a lo largo del cual luchó por despojarse de las formas de pensamiento que lo habían constituido intelectualmente para "tojolabalizarse", para integrarse al mundo comunitario del "nosotros"; en fin, para ser reconocido por los indígenas como el hermano Carlos.

Entonces a petición de los propios indígenas y del obispo organizó un equipo de setenta colaboradores, originarios de diversas comunidades, para traducir el Nuevo Testamento a partir de los textos griegos. Un número de traductores que de inmediato evoca la elaboración precristiana de la versión griega de la Biblia. En el caso actual se pretendía, más allá del traslado fiel del sentido de los escritos, la creación de una versión desde la óptica tojolabal. Años después, Carlos recordaba las expresiones de desacuerdo de los traductores con las ideas del Apóstol de los Gentiles, mientras que el Apocalipsis era aceptado sin dificultad, por reconocerlo como un relato de símbolos, visiones y sueños; temas familiares para la cosmovisión maya. Cinco años de trabajo dieron por resultado la obra: ja yajk'achil sju' unil ja dyosi b'a tojol ab'al, editada en Comitán por La Castalia y aparecida en dos volúmenes (uno en 1991 y el otro en 1993).

El levantamiento zapatista sorprendió a Carlos en la ciudad de México. Advertido por el obispo del peligro que corría de ser deportado, en momentos cuando se acusaba a los agentes de la pastoral diocesana de haber fomentado y hasta organizado la rebelión, ya no pudo regresar. Aunque el riesgo no le impidió reanudar, en cuanto fue posible, la comunicación con los tojolabales y tiempo después volver a Chiapas en distintas ocasiones. Ni la búsqueda de responsables de la sedición por parte del gobierno, ni la calidad burocrática de extranjero que siempre mantuvo, lo volvieron prudente en cuanto a la expresión de sus denuncias acerca de los abusos cometidos por "los mandones" contra la población indígena.

En las circunstancias inciertas de 1994, el Centro de Estudios Mayas, entonces coordinado por Ana Luisa Izquierdo, le abrió al doctor Lenkersdorf de nuevo las puertas de la UNAM, para que se integrara como investigador titular en el campo 
de la linguiística, en el Instituto de Investigaciones Filológicas. Resulta pertinente recordar que ni Carlos ni los tojolabales eran ajenos a este centro académico. Desde fines de los setenta, a instancias del linguiista Otto Schumann y bajo la coordinación de Mario $\mathrm{H}$. Ruz, se había realizado un proyecto de carácter multidisciplinario, cuyos resultados se dieron a conocer en cuatro volúmenes titulados Los legítimos hombres. Aproximación antropológica al grupo tojolabal, publicados en 1981, 1982, 1983 y 1986. En el último aparecía uno de los primeros trabajos de investigación histórica de Gudrun Lenkersdorf. Además, hacia principios de los noventa, el propio Carlos había empezado a publicar algunos artículos en la revista Estudios de Cultura Maya, órgano de difusión del mismo centro. Ya como investigador su primer libro coeditado por la unAm y Siglo XXI Editores en 1996 fue: Los hombres verdaderos. Voces y testimonios tojolabales. Lengua y sociedad, naturaleza y cultura, artes y comunidad cósmica. La obra había recibido en 1994 el Premio de Ensayo Literario Hispanoamericano Lya Kostakowsky, y al presente ha alcanzado cuatro ediciones, además de haber sido traducida al francés.

Desde que regresó a México, con casi setenta años a cuestas, Carlos realizó una actividad incesante, encaminada a difundir su interpretación de la cultura maya en la versión tojolabal, por medio de conferencias impartidas en diversas instituciones de la República Mexicana y de Guatemala, Alemania, Bélgica, Italia, Israel y los Estados Unidos, y a través de la redacción de un gran número de artículos y capítulos de libros editados tanto en nuestro país como en el extranjero. En cuanto a la publicación de libros, unos fueron resultado de su coordinación, como: Indios somos con orgullo. Poesía maya-tojolabal (1999, 2004), otros de su trabajo editorial: El diario de un tojolabal. Edición bilingüe, traducción y comentarios (2001), además de las obras de su autoría individual. Entre los libros que escribió en español se cuentan: Tojolabal para principiantes. Lengua y cosmovisión mayas en Chiapas (1994, 2002), Filosofar en clave tojolabal (2002), Conceptos tojolabales de filosofía y del altermundo (2004), La semántica del tojolabal y su cosmovisión (2006), y el que recoge sus últimas reflexiones: Aprender a escuchar: enseñanzas mayatojolabales (2008).

Una actividad que llenó de satisfacción los últimos años de Carlos, cuando se enfrentaba con valentía y un optimismo envidiable al cáncer, fue la docencia en la Facultad de Filosofía y Letras de la unAm, donde impartió cursos de lengua y cultura tojolabal destinados a los alumnos de licenciatura y posgrado en los programas de Filosofía, Estudios Latinoamericanos y Mesoamericanos, pero abiertos a todos los interesados. Los jóvenes educados en la desesperanza o en la autocomplacencia de la llamada posmodernidad abarrotaban el salón de clases para escuchar sentados hasta en el suelo al anciano maestro que, con voz frágil, les anunciaba, por medio de anécdotas de su vida entre los tojolabales, la buena nueva de la existencia de una sociedad basada en relaciones horizontales de respeto mutuo y verdadera democracia, capaz de vivir en armonía con la naturaleza y ajena al egoísmo individualista. Al mismo tiempo les despertaba la conciencia respecto de la necesidad de comprometerse con la construcción de 
una sociedad nacional más equitativa y tolerante. Inspirados por sus palabras, todos terminaban cantando las canciones compuestas por los indios que lo eran con orgullo. En agosto de 2010, Carlos se despidió de los estudiantes, pues aunque su mente se mantenía lúcida y combativa, la enfermedad había avanzado a tal grado que le impedía continuar con la que asumió como una misión. Tres meses después, ataviado con la camisa que las tojolabales le habían bordado y portando el bastón de autoridad que algún día le entregó una comunidad, Carlos emprendió la última jornada. En manos de sus discípulos queda la tarea de trabajar para convertir en realidad la utopía.

Además de colegas en el Centro de Estudios Mayas, Gudrun y Carlos son mis amigos y vecinos. A los dos les agradezco la generosidad que han mostrado, a lo largo de los años, al haberme hecho partícipe de sus recuerdos.

María del Carmen León Cázares Universidad Nacional Autónoma de México, Instituto de Investigaciones Filológicas,

Centro de Estudios Mayas 